\section{Usuários de drogas injetáveis e infecção pelo Vírus da Imunodeficiência Humana: epidemiologia e perspectivas de intervenção}

\section{Injecting drug users and the Human Immunodeficiency Virus infection: epidemiology and perspectives of intervention}

\author{
Waleska Teixeira Caiaffa \\ Departamento de Medicina Preventiva e Social \\ Faculdade de Medicina UFMG \\ Av. Alfredo Balena, 190 - Caixa Postal 340 \\ 30130-100 Belo Horizonte, MG, Brasil \\ e-mail:wcaiaffa@medicina.ufmg.br
}

\section{Francisco Inácio Bastos}

Departamento de Informações em Saúde FIOCRUZ

Av. Brasil, 4365

21042-900 Rio de Janeiro, RJ, Brasil.

e-mail:bastos@fiocruz.br

\section{Resumo}

Dos cerca de 130 mil casos de aids notificados no Brasil entre 1980 e fevereiro de 1998, 21\% estão diretamente relacionados ao uso de drogas injetáveis. Este artigo revisa aspectos da epidemiologia da infecção pelo HIV e outros patógenos de transmissão sangüínea, entre usuários de drogas injetáveis (UDI), visando a subsidiar as pesquisas e a implementação de propostas de intervenção em nosso meio. A população de UDI é heterogênea e, ao contrário do estereótipo, nem todos os UDI provêm de minorias masculinas das grandes áreas urbanas. Esta população está em contínua transformação, com mudanças marcantes ao longo do tempo na sua composição, hábitos e padrões de consumo. A estimativa precisa do número de UDI em uma dada localidade constitui tarefa difícil, embora disponha-se de novas metodologias, como o método de captura-recaptura. A combinação de dados provenientes de diversas fontes se mostra também indispensável. Estudos no sentido de monitorizar os padrões e tendências do uso de drogas injetáveis, segundo diferentes níveis de agregação e em intervalos regulares, permitem estimar prováveis aumentos ou decréscimos desta população e detectar mudanças relevantes nos seus comportamentos, contribuindo para orientar as estratégias de intervenção e alocação ótima de recursos. Evidências recentes demonstram que, devido à exposição parenteral e sexual aos diferentes patógenos, as infecções nesta população ocorrem precocemente, demandando intervenções prontas e abrangentes quanto aos UDI em atividade, e esforços no sentido de minimizar a transição para a via injetável e oferecimento de alternativas de tratamento clínico e para o abuso de drogas.

Palavras-chave: Abuso de substâncias por via endovenosa. Uso comum de agulhas e seringas. HIV. Vírus da hepatite. Vírus HTVL-I e II. Síndrome de imunodeficiência adquirida. Epidemiologia. 


\section{Abstract}

As of February, 1998 about 130 thousand AIDS cases were reported in Brazil; $21 \%$ of them among injecting drug users (IDUs). The present paper reviews the main findings of the HIV epidemiology and other blood-borne pathogens infection among IDUs, in Brazil and abroad, to foster the ongoing research and preventive initiatives carried out in Brazil. Data have shown that, far from the stereotype of a male population living in the main metropoli$\tan$ areas, IDU population is rather heterogeneous. This population is under continuous turnover, with relevant changes in its very composition, habits and drug consume patterns. An accurate estimation of the size of IDU population in different settings and places is a puzzling task, although recent methodologies, e.g. capture-recapture methods, seem to be a reliable strategy. The use of combined data from different sources is also very useful in the process of population size estimation. Comprehensive researches carried out in different settings, using data from distinct and complementary aggregation levels, under regular follow-up, are essential to monitor patterns and trends of the drug users population and drug consume patterns, especially the injection of illicit drugs. These data are essential to the implementation and permanent evaluation of preventive strategies, as well as tools for the optimal allocation of scarce resources. Due to the double risks to which IDUs are exposed - parenteral and sexual - different infections are frequent in this population, taking place in an early moment of their drug use "career". Successful interventions must be precocious and comprehensive, providing counseling, testing for different pathogens, and access to condoms and sterile injection equipment. Another core interventions set involves the prevention of the transition from non-injecting to injecting routes, and efforts to offer low-threshold and available anonymous treatment at no cost for either their clinical problems and harms secondary to drug abuse.

Keywords: Substance abuse, intravenous. Needle sharing. HIV. Hepatitis viruses. HTVLI and II BLV viruses. Acquired Immunodeficiency Syndrome. Epidemiology.
A epidemia da síndrome da imunodeficiência adquirida (aids) entre os usuários de drogas injetáveis no Brasil e no mundo e as estratégias preventivas

No Brasil, dos 128.821 casos de aids notificados ao Ministério da Saúde no período de 1980 a fevereiro de 1998, 21\% estão diretamente relacionados ao uso de drogas injetáveis. ${ }^{1}$ Ainda que em anos recentes a epidemia pelo HIV (Human Immunodeficiency Virus) / aids entre usuários de drogas injetáveis (UDI) tenha, aparentemente, reduzido a sua velocidade de expansão $0^{2,3}$, esta via de transmissão continua sendo um fator essencial na alteração contínua do perfil da epidemia, tanto no sentido de abranger novos estratos sociais (via de regra, mais pobres e marginalizados), como novas regiões geográficas. ${ }^{4}$

Para efeito de comparação, nos Estados Unidos, até dezembro de 1997, aproximadamente 641.086 casos de aids em adultos foram notificados e cerca de $36 \%$ estavam relacionados direta ou indiretamente ao uso de drogas injetáveis. ${ }^{5}$ Em contraste, na Austrália, onde medidas de controle da epidemia entre os UDI têm sido adotadas desde 1985, mesmo antes de uma compreensão melhor da dinâmica da epidemia entre os UDI, cerca de $2,5 \%$ do número de casos de aids foram atribuídos aos UDI em $1994 .{ }^{6}$

Na Tabela, podem ser observados os coeficientes de incidência dos casos de aids notificados no Brasil, Estados Unidos e Austrália de 1982 a 1993. O Brasil situa-se numa posição intermediária entre os outros dois países. Nota-se que o Brasil apresenta um perfil semelhante aos Estados Unidos, com uma tendência crescente dos coeficientes a partir de 1988-9, o mesmo não sendo tão evidente na Austrália, onde os coeficientes de incidência dos casos notificados permanecem relativamente estáveis em torno de 45 casos por um milhão de habitantes, desde $1991^{7}$. Estudos sugerem a participação importante dos UDI na epidemia de aids a partir do final da década de 80, tanto nos Estados Unidos como no Brasil. 
Tabela - Coeficiente de incidência dos casos de aids notificados (por milhão) na Austrália, Brasil e Estados Unidos, $1982-1993$.

Table - Incidence of reported AIDS cases (per million) in Australia, Brazil and United States, 1982-1993.

\begin{tabular}{|c|c|c|c|c|c|c|c|c|c|c|c|c|}
\hline País & 1982 & 1983 & 1984 & 1985 & 1986 & 1987 & 1988 & 1989 & 1990 & 1991 & 1992 & 1993 \\
\hline Austrália & 0,1 & 0,3 & 2,6 & 7,1 & 14,5 & 23,7 & 31,9 & 36,0 & 38,6 & 45,8 & 43,9 & 44,1 \\
\hline Brasil* & 0,0 & 0,0 & 1,0 & 4,0 & 8,0 & 19,0 & 30,0 & 41,0 & 56,0 & 75,0 & 92,0 & 104,0 \\
\hline Estados Unidos & 6,0 & 12,0 & 24,0 & 49,0 & 74,0 & 111,0 & 138,0 & 165,0 & 185,0 & 224,0 & 285,0 & 276,0 \\
\hline
\end{tabular}

* Até fevereiro de 1998, o coeficiente de incidência dos casos notificados de AIDS foi de 923/1 milhão (Região Sudeste: 1520/1 milhão).

Fonte: Adaptado de Feachem ${ }^{7}$ (1995).

* As of February 1998, the incidence rate of reported AIDS cases was 923/million (The Southeast region: 1,520/million).

Source: Adapted from Feachem ${ }^{7}$ (1995).

Um exemplo recente da importância da participação dos usuários de droga injetável na epidemia tem sido a explosão, nos últimos três anos, das taxas de infecção pelo HIV em países da antiga União Soviética. Na Ucrânia, o país mais afetado, foi registrado um aumento de cerca de 30 vezes no número de infecções entre 1995 e 1997, sendo a maioria relacionada ao uso indevido de drogas. Quatro, de cada cinco indivíduos recentemente diagnosticados com a infecção pelo HIV, são usuários de droga injetável naquele país. ${ }^{8}$

Modelos hierárquicos relacionados ao uso de drogas e estratégias para prevenir a transmissão parenteral do HIV entre UDI têm sido propostos ${ }^{9}$. A estratégia tradicional tem sido a abstinência de drogas. No entanto, apesar das taxas de infecção pelo HIV entre os UDI que freqüentam programas de tratamento do abuso de drogas serem aparentemente menores, quando comparados com aqueles que não os freqüentam ${ }^{10-13}$, somente pequeno percentual de UDI se encontra em tratamento em um determinado momento, com estimativas que variam de $6 \%$ a $22 \%^{13,14}$. Portanto, embora o tratamento represente uma importante estratégia, não é realista considerar que somente a expansão desta estratégia conseguirá conter a infecção pelo HIV entre os UDI.

\section{Epidemiologia do uso de drogas injetáveis e do HIV/aids entre UDI}

A estimativa do número de UDI em uma população representa uma importante e desafiadora tarefa, principalmente para o planejamento das estratégias relativas ao enfrentamento da epidemia pelo HIV/aids, além de outras patologias de transmissão sangüínea, como as hepatites virais e o vírus humano T-linfotrópico, tipos I e II (HTLV I e II). Tanto em nível federal, quanto estadual e municipal, os profissionais de saúde necessitam de uma estimativa precisa do tamanho, das características e da distribuição geográfica da população de UDI, objetivando uma alocação de recursos racional e adequada. Igualmente, conhecer em detalhe o perfil epidemiológico dos UDI é fundamental para que se possa estabelecer a "situação de base" (baseline) deste grupo, de modo a possibilitar posteriores estudos de efetividade das medidas de intervenção.

Os padrões de consumo de drogas injetáveis variam com relação à natureza $\mathrm{e}$ às combinações das drogas utilizadas, à freqüência de uso, às vias de auto-administração, à freqüência de compartilhamento dos equipamentos, entre outros fatores, além de um conjunto de variáveis da esfera da psicologia individual e grupal, e das condições socioeconômicas e culturais. No Brasil, por exemplo, um estudo multicêntrico mostrou que a droga mais utilizada entre UDI foi a cocaína, consumida por 94 a $100 \%$ dos UDI entrevistados, o que confirma estudos anteriores desenvolvidos em localidades específicas, como o Rio de Janeiro ou Santos ${ }^{15-17}$. Em outros países como os EUA e Austrália, a heroína detém uma parcela importante do uso injetável, com $20 \%$ e $47 \%$, respectivamente ${ }^{6,14}$. Também o uso combinado de drogas, dependendo da disponibilidade das diferentes substâncias no mercado, é bastante comum em diversos locais.

A forma e freqüência com que os UDI usam as drogas também é particularmente relevante porque usuários de cocaína tendem a injetar em "binges" ou "porres" ou "baques", caracterizados pela injeção de 
doses repetidas em curtos intervalos de tempo, alternando uma alta intensidade de uso com intervalos longos de não consu$\mathrm{mo}^{18}$. Usuários de heroína, ao contrário, tendem a injetar de uma maneira mais regular, desde poucas injeções ao dia até injeções regulares ao longo de vários dias num período mais longo. Os usuários de cocaína podem, por isso, ser mais suscetíveis aos riscos decorrentes do compartilhamento de seringas, devido à necessidade de administrações repetidas da droga para evitar os efeitos depressivos que se seguem à intoxicação pela droga ${ }^{19}$.

Enumerar simplesmente o número de UDI a que se tem acesso, embora possa fornecer alguma idéia da magnitude do problema do uso de drogas, contribui pouco para atingir alguns objetivos tais como conhecer a possível associação entre determinados perfis de consumo de droga e a dinâmica de transmissão do HIV e outros patógenos de transmissão sangüínea. De fato, em um estudo da associação entre o hábito de injetar cocaína e a infecção pelo HIV, 26,4\% dos usuários de cocaína eram soropositivos para o HIV quando comparados com $10,6 \%$ dos usuários de outras drogas. Ou seja, usuários de cocaína apresentavam um risco três vezes maior de estar infectados pelo HIV. ${ }^{19}$

Portanto, conhecer a população de UDI, comparar o segmento de UDI a que se tem acesso com outras populações e determinar o perfil do consumo de drogas podem contribuir de maneira importante para o conhecimento de medidas preventivas a adotar em relação à epidemia do HIV/aids.

\section{Estimativas do tamanho da população de UDI}

Dada a extraordinária dificuldade de se estimar a população de UDI, decorrente do caráter ilegal deste comportamento e da marginalização e estigmatização desta população, alguns autores têm proposto modelos matemáticos baseados em técnicas de estimativa de prevalência, para estimar a população de UDI.
Estima-se que mais de cinco milhões de pessoas utilizariam drogas injetáveis no mundo ${ }^{8}$. Estimativas do número de UDI no Brasil são inexistentes. Nos Estados Unidos, foi estimado em cerca de 500.000 a 2.000 .000 o número de UDI em todo o país. A partir de premissas de que: (1) $85 \%$ dos UDI residem em áreas metropolitanas; (2) o número total de UDI é cerca de 2,5 a 20 vezes maior do que o número de UDI em tratamento, e cerca de 2,9 e 10,5 vezes maior em relação ao número de UDI testados para o HIV, foi estimado que o número total de UDI "em atividade" nos Estado Unidos situa-se em torno de 1,7 milhões de indivíduos. No entanto, tais estimativas podem ser imprecisas, recomendando-se seu uso com precaução. ${ }^{20}$

Recentemente, uma técnica no campo da biologia, denominada captura-recaptura, usada inicialmente para estimar a densidade de uma população de animais silvestres tem sido utilizada como um método complementar ou alternativo à estratégia tradicional da simples enumeração de uma população, principalmente as de difícil acesso, como a dos usuários de droga. Assim, Drucker e Vermund ${ }^{21}$, Abeni e col. ${ }^{22}$ e Mastro e col. ${ }^{23}$, usando sistemas de informação diversos de detecção da infecção pelo HIV, puderam estimar a população de UDI respectivamente no Bronx, Nova Iorque; em Lazio (Itália) e em Bangkok (Tailândia). No Brasil, resultados preliminares do uso desta técnica em estudo de prevalência neste grupo parecem promissores ${ }^{24}$.

Inquéritos populacionais sobre o uso e abuso de drogas podem eventualmente constituir outra fonte de informação para estimar o tamanho e perfil da população de UDI. Tais estratégias, no entanto, têm-se mostrado bastante limitadas. No que se refere à detecção dos usuários "pesados", cuja interação com as equipes de pesquisa e mesmo com o sistema formal de saúde é complexo, diversas dificuldades logísticas são encontradas. $\mathrm{O}$ acesso a estes indivíduos é muitas vezes impedido pela ausência de endereços fixos, a qualidade da informação obtida é, com freqüência, questionável, o que, somando-se à estigmatização destes 
comportamentos, pode culminar na recusa de participar ou de fornecer tais informações. Tais problemas geralmente acarretam um diferencial da informação em favor daqueles que não respondem ou respondem negativamente ao uso de drogas (subnotificação e/ou subestimação da gravidade dos padrões de consumo).

No Brasil, apesar da última Pesquisa Nacional sobre Demografia e Saúde (PNDS) ${ }^{25}$ contemplar questões ligadas à aids, não incluiu quesitos referentes ao uso e abuso de drogas lícitas e ilícitas. Já os inquéritos sobre o uso de tabaco, álcool e drogas lícitas e ilícitas em jovens estudantes brasileiros, coordenado pelo Centro Brasileiro de Informações sobre Drogas Psicotrópicas (CEBRID), realizados nos anos de 1987, 1989 e 1993, representam um dos conjuntos de dados mais abrangentes sobre o consumo de drogas no Brasil ${ }^{26-29}$. No entanto, estes inquéritos sofrem da mesma limitação anterior quanto à capacidade de estimar os usuários "pesados", representados principalmente por aqueles que injetam drogas, e que freqüentemente iniciam este padrão de consumo específico após um longo período de consumo por outras vias quando, de um modo geral já estão fora da escola $^{30}$.

Embora, até o momento não tenha sido relatado o uso injetável de drogas por estes inquéritos, os resultados do último levantamento, feito em 1993, revelam um percentual de $26 \%$ para o uso na vida de todas as drogas pesquisadas, exceto para o tabaco e álcool, cujos percentuais foram de $37 \%$ e 85\%, respectivamente. É importante ressaltar o elevado percentual de escolares que relataram o uso freqüente de álcool (24\%). Em termos de tendência temporal, estes estudos indicam um crescimento do uso na vida de maconha, anticolinérgicos e cocaína no período compreendido entre 1987 e $1993^{29}$.

Mesmo estudos desenvolvidos entre UDI, baseados em amostras de tamanho apropriado, independentemente do vínculo ou não destes com centros de tratamento, apesar de oferecerem informações relevantes a respeito de características dos
UDI podem apresentar limitações devido à questão de bias ou viés de seleção ou voluntariado. Ou seja, UDI que se dispõem a fornecer tais informações podem ou não ser semelhantes ao conjunto da população de onde provêm. Portanto, a obtenção de uma amostra realmente representativa dos UDI é extremamente difícil ou quase impossível. A estratégia mais recomendada no sentido de fornecer dados que possibilitem alguma generalização é composta de esquemas múltiplos de amostragem, incluindo os UDI que recém-ingressaram em programas de tratamento, aqueles inseridos no sistema jurídico-penitenciário e aqueles recrutados na comunidade. Comparações acerca dos padrões diversos de comportamentos de risco e das taxas de infecção para o HIV e outras doenças veiculadas pelo sangue possibilitam validar estimativas e estabelecer inferências.

\section{Perfil dos UDI}

Ao contrário do pensamento inicial, tributário do senso comum, as análises da distribuição geográfica e das características sociodemográficas e de consumo de drogas dos UDI têm revelado uma grande diversidade dos indivíduos reunidos sob esta denominação comum.

\section{Distribuição geográfica dos UDI}

Os UDI parecem se agrupar em determinadas macroáreas geográficas, embora estes agrupamentos possam se revelar bastante dispersos a partir de uma análise mais pormenorizada e de menor nível de agregação. No Brasil, os casos de aids atribuídos aos UDI parecem se agrupar em determinados "corredores" ligados ao tráfico de drogas, tais como os portos marítimos - Santos, SP ou Itajaí, SC; ou regiões que se supõem atravessadas pela rota das drogas, tais como Cuiabá, a região Sul e o Triângulo de Minas Gerais.

A epidemia pelo HIV/aids entre UDI, no Brasil, concentrou-se inicialmente de forma clara numa faixa no sentido leste-oes- 
te, conectando o Centro-oeste ao litoral paulista, através do Estado de São Paulo ${ }^{31}$. Já a tendência mais recente, tanto dos casos notificados de aids como dos estudos de soroprevalência (ressaltando a importante defasagem temporal das informações captadas por uns e outros) é de uma presença crescente do litoral sul. ${ }^{4}$

\section{Características sociodemográficas e padrões de consumo de drogas}

O estudo feito pelo NADR (NIDA's National AIDS Demonstration Research), realizado em cerca de 13.000 UDI norteamericanos, em 28 diferentes cidades, oferece o perfil mais fidedigno de UDI em atividade, mas não vinculados a centros de tratamento para o abuso de drogas. A idade média destes UDI situava-se entre 31 a 40 anos, e estes indivíduos relatavam o uso de drogas por um período de 10 a 19 anos. De 9 a 95\% dos UDI, dependendo do local em que se obteve a amostra, eram negros (51\% para todo o grupo); de 0 a $81 \%$ eram de origem latina (25\% para todo grupo) e, de 3 a $65 \%$ eram brancos (22\% para todo grupo). A proporção de mulheres era de 12 a $37 \%$ ( $26 \%$ para todo grupo). Cerca de 31 a $67 \%$ dos UDI tinham $2^{\circ}$ grau completo (45\% para todo grupo), e 20 a $75 \%$ relatavam estar desempregados (55\% para todo grupo); $81 \%$ relatavam ter sido presos alguma vez (intervalo: 29 a 75\%), e $59 \%$ haviam sido atendidos em centros de tratamento para abuso de drogas (intervalo: 29 a $75 \%)^{32}$.

Quanto ao perfil do consumo, a principal droga injetada foi a heroína $(28 \%)$, seguida pela cocaína $(21 \%)$ e pela combinação de ambas - speedball (35\%), com enorme variação entre os diferentes locais. Com relação ao compartilhamento, $68 \%$ compartilhavam com amigos, $67 \%$ com conhecidos, $52 \%$ com parceiros e $25 \%$ com estranhos $^{32}$.

Como comparação, podem-se usar os resultados preliminares do Projeto Brasil, o primeiro estudo multicêntrico nacional realizado na população de UDI ${ }^{15}$, precedido apenas por estudos desenvolvidos nas cidades do Rio de Janeiro ${ }^{16}$ e Santos ${ }^{17}$, no âmbito do Estudo Multicêntrico da Organização Mundial da Saúde (WHO International Collaborative Group ${ }^{33}$, 1994). Este inquérito epidemiológico multicêntrico foi desenvolvido entre 1994 e 1996, em uma amostra de 631 UDI. A faixa etária destes UDI situou-se em torno de 16 a 55 anos, e a idade do início do uso de drogas variou entre 17 e 19 anos. A droga mais consumida pela via injetável foi a cocaína (94 a 100\%); com relevante consumo concomitante de cocaína por via não injetável, tanto cheirada, como sob a forma de $\operatorname{crack}$ (36 a $61 \%$ ), além do relato do uso de heroína por via injetável ( 0 a 17\%), esta última, droga recentemente introduzida no Brasil e de presença discutível na "cena de consumo" brasileira, exceção feita ao segmento específico de usuários mais graves ${ }^{34}$. O compartilhamento foi relatado por 56 a $85 \%$ dos entrevistados, dependendo da cidade onde a amostra foi recrutada. Observou-se uma alta prevalência para o HIV (28 a 71\%). Tais dados corroboram as observações internacionais acerca da diversidade do perfil dos usuários de drogas.

\section{Disseminação do HIV/aids entre UDI}

Os coeficientes de prevalência e incidência do HIV entre UDI variam consideravelmente, dependendo da localização geográfica. Hahn e col..$^{35}$, revisando 98 estudos sobre UDI em centros de tratamento, relatam uma soro-prevalência de 0 a $65 \%$ nos Estados Unidos. No Brasil, os coeficientes de prevalência variam entre $16 \%$ e $74 \%$ em amostras de UDI em centros de tratamento, com prevalências bastante elevadas - $66 \%$ - de infecção entre usuários basicamente recrutados nas "ruas" em Santos, $\mathrm{SP}^{3}$.

Ainda que alguns estudos nesta população tenham encontrado taxas de infecção similares, ao longo do tempo a partir de estudos seccionais seriados, não resta dúvida de que, uma vez que a infecção pelo HIV tenha se estabelecido numa comunidade de UDI (diga-se com uma prevalên- 
cia maior do que $10 \%$ ), os coeficientes de prevalência subseqüentes tendem a aumentar dramaticamente nos próximos dois a quatro anos, na ausência de programas de prevenção. ${ }^{9}$

Os coeficientes de incidência entre UDI que freqüentam programas de tratamento variam de 0 a $14 \%$ nos Estados Unidos. Com relação aos UDI fora destes programas, as taxas de incidência da infecção pelo HIV permanecem altas em alguns locais, com cerca de quatro novas infecções por 100 pessoas-ano de observação ${ }^{36}$.

A eficiência da transmissão entre UDI varia de acordo com os diversos comportamentos de risco deste segmento. São eles: freqüência de injeção e de compartilhamento dos equipamentos usados, número de parceiros com quem compartilham estes equipamentos e práticas sexuais. Vários estudos apontam para o compartilhamento como o fator de risco mais importante quanto à soro-conversão e a determinação de altos coeficientes de prevalência do HIV.

Alguns fatores demográficos também estão associados à infecção. De uma maneira geral, as taxas tendem a ser maiores entre UDI que pertencem a minorias étnicas e /ou culturais, mulheres e usuários iniciantes e mais jovens ${ }^{37,38}$.

\section{Transmissão sexual entre UDI}

A transmissão sexual dos UDI para seus parceiros representa um risco para homens e mulheres. Além disto, a chance de um UDI ter um parceiro que também faz uso de drogas injetáveis é elevada, particularmente entre as mulheres. Estima-se que 75 a 90\% das mulheres UDI têm parceiros que consomem drogas por esta via, e que de 20 a $50 \%$ dos homens UDI têm parceiras $\mathrm{UDI}^{39}$. A literatura cita com razoável constância o fato de parceiras sexuais de UDI do sexo masculino utilizarem o sexo como uma estratégia para obter drogas, ao mesmo tempo em que compartilham com seus parceiros os equipamentos de injeção, expondo-se, portanto, a duas fontes de exposição. Estas mulheres tendem também a não usar preservativos, o que aumenta estes riscos ${ }^{40}$. Igualmente observa-se que as usuárias de crack, independentemente do fato de serem ou não UDI, "trocam sexo por droga”. Este comportamento pode servir de ponte entre o hábito de injetar drogas e o risco de contaminação heterossexual ${ }^{41}$.

Além disto, devido à intensa "desinibição" produzida pelo crack, as atitudes de prevenção, tais como o uso de preservativos ou de equipamentos de injeção estéreis, são negligenciadas. Um fator adicional é o papel do crack na disseminação da sífilis por mecanismos similares, o que por seu turno pode facilitar a transmissão do HIV, devido à maior susceptibilidade dos pacientes portadores de sífilis e outras DST à infecção pelo HIV ${ }^{42}$.

Diversos estudos demonstram que os programas de redução de dano têm relativamente maior sucesso em influenciar seus participantes, no sentido de alterar seus comportamentos de risco com relação ao hábito de injetar do que em relação aos comportamentos sexuais de risco ${ }^{43}$. Com relação às mulheres, isto é particularmente difícil, uma vez que as mudanças no sentido de práticas sexuais mais seguras esbarram, com freqüência, na falta de cooperação e na resistência dos parceiros ${ }^{44}$.

A adequada classificação quanto à natureza da transmissão do HIV entre UDI constitui uma questão difícil. Muitas vezes, a infecção atribuída à transmissão parenteral pode ser secundária à interação sexual desprotegida. Alguns autores mencionam mesmo um processo de subestimação sistemática do real papel da transmissão sexual entre UDI ${ }^{45}$. Aventa-se também a hipótese de que os UDI poderiam ser mais infectantes do que indivíduos portadores do HIV pertencentes a outras categorias de exposição, existindo ainda a hipótese alternativa de que seus parceiros sexuais poderiam ser mais susceptíveis. Padian e col. ${ }^{46}$, por exemplo, relatam taxas mais elevadas de transmissão do HIV do homem para a mulher entre UDI frente aos não-UDI, embora não haja clareza do ponto de vista biológico de quais os motivos que determinariam este diferencial de transmissibilidade. 


\section{Transmissão vertical entre mulheres UDI}

Vários estudos apontam para a elevada freqüência de recém-nascidos infectados pelo HIV, filhos de mulheres UDI ${ }^{47}$. Mulheres UDI parecem engravidar com maior freqüência do que as não usuárias de droga injetável, fato atribuído ao uso inconsistente de preservativos, ou simplesmente à não utilização destes. Um estudo de Selwyn e col. ${ }^{48}$, entre mulheres UDI, revelou que $24 \%$ das mulheres HIV soropositivas engravidaram ao longo do período de seguimento no âmbito de um programa de tratamento, para uma proporção de $22 \%$ dentre as mulheres UDI não infectadas pelo HIV. O mesmo estudo mostrou que um número similar das gestantes, infectadas ou não, optaram pela interrupção da gravidez ${ }^{48}$.

\section{Transmissão de outras doenças veiculadas pelo sangue entre UDI e coinfecções}

Os UDI estão igualmente sujeitos ao risco de se infectar por outros patógenos de transmissão sangüínea que não o HIV, o que inclui o vírus da hepatite $\mathrm{C}$ (VHC), da hepatite B (VHB) e o vírus humano Tlinfotrópico, tipos I e II (HTLV-I/II). A transmissão destes agentes infecciosos entre UDI é primariamente parenteral, através do compartilhamento dos equipamentos de injeção.

Estudos recentes têm mostrado que os coeficientes de soroprevalência de infecção por estes patógenos são relativamente altas entre UDI, e diretamente correlacionadas ao tempo do uso injetável de drogas. Assim, em um estudo de coorte em Baltimore, Estados Unidos, Garfein e col. ${ }^{49}$, através de uma análise transversal usando dados da linha de base, mostram que entre UDI que utilizaram drogas injetáveis por um período de 4 a 5 anos, os coeficientes de soroprevalência eram as seguintes: $85,0 \%$ para o VHC; $77,4 \%$ para o VHB; $28,0 \%$ para o HIV e 2,1\% para o HTLV. Taxas bastante similares foram encontradas em outra coorte de UDI, cujo tempo médio de exposição às drogas inje- táveis era de 12 anos. Utilizando as taxas de infecção para o VHC e VHB, estes autores concluíram que a maioria das novas infecções ocorreriam logo após o início do uso injetável de drogas, estando basicamente associadas às práticas de risco da injeção e não às práticas sexuais. Encontraram ainda uma alta prevalência de portadores crônicos de VHC (50 a 90\%) e VHB (5 a 10\%). Para o VHC e VHB as taxas de infecção se elevaram acentuadamente já no primeiro ano de uso injetável, sugerindo uma alta eficiência da transmissão parenteral ${ }^{49}$.

Já para o HIV, a trajetória de aumento nos dois primeiros anos de injeção foi gradual, sugerindo que o HIV não se dissemina tão eficazmente quanto o VHC e VHB. Neste último caso, as variáveis "ser solteiro" e "ter orientação sexual diferente da heterossexual" se mostraram associadas à infecção pelo HIV, sugerindo que o HIV poderia ser mais eficientemente transmitido pela via sexual nesta população do que o VHC e o $\mathrm{VHB}^{49}$.

Em suma, estes dados sugerem que os UDI estariam sob maior risco de se infectar por patógenos de transmissão sangüínea, como o VHB e o VHC, logo no início de suas práticas injetáveis, reforçando a idéia de se desenhar programas de intervenção especificamente dirigidos aos que se iniciam no hábito de injetar e àqueles sob risco de passarem de vias não-injetáveis para vias injetáveis de consumo de drogas ${ }^{50,51}$. Assim, tanto a prevenção primária quanto a secundária do uso de drogas devem ser enfatizadas.

O uso da infecção pelo VHC e VHB como marcadores de efetividade de programas de intervenção, objetivando a redução do risco de se infectar pelo HIV, deve ser bem avaliado, no sentido de se determinar qual o momento para se proceder a este conjunto de medidas, levando-se em consideração que a maioria dos UDI têm maior chance de converter sorologicamente para estes dois agentes infecciosos logo no primeiro ano da prática de se injetar. Finalmente, intervenções visando a reduzir a infecção pelo HIV devem contemplar o comportamento sexual de risco, tanto quanto as práticas de injeção. 
Em resumo, cada UDI infectado pode dar lugar a uma série de novas infecções, não só pelo HIV como por outras doenças veiculadas pelo sangue como as hepatites virais B, C, e a infecção pelo HTLV-I e II e seus respectivos quadros clínicos.

As evidências de que o uso compartilhado de drogas injetáveis desempenha um papel relevante na dinâmica subseqüente da epidemia pelo HIV entre mulheres e crianças são inúmeras, inclusive em estudos ecológicos nacionais ${ }^{52}$, fazendo com que a redução nas taxas de infecção pelo HIV entre os UDI acarrete uma redução destas taxas em seus parceiros sexuais e futuros conceptos.

Relevante na avaliação da efetividade dos programas de redução de danos é o achado consistente de que o compartilhamento de seringas é responsável por proporção substancial de novas infecções pelo HIV entre os UDI. Promover a distribuição de seringas estéreis e/ou a troca de seringas usadas por seringas novas, ou, ainda que com menor eficácia ${ }^{53}$, a adequada desinfecção de seringas constitui estratégia fundamental na redução da infecção pelo HIV nesta população.

Cabe observar, no entanto, que outras práticas direta ou indiretamente relacionadas ao uso indevido de drogas, tais como as práticas sexuais de risco, são capazes de perpetuar a transmissão do HIV nesta população, e parecem sofrer menor impacto dos programas de redução de danos, ainda quando estes reúnem um conjunto abrangente de iniciativas preventivas ${ }^{45}$. Por essa razão, a avaliação dos programas de redução de danos que utiliza exclusivamente a soroconversão para o HIV como marcador da sua efetividade provavelmente apresentará limitações relevantes a médio prazo. Programas de redução abrangentes, contemplando de forma sistemática e específica a prevenção da transmissão sexual nesta população, provavelmente terão resultados mais satisfatórios, ainda que como mencionado acima, longe do ideal, não só com relação ao HIV, mas também com relação aos demais patógenos veiculados pelo sangue e/ou pelas relações sexuais.

\section{Como monitorizar as tendências da epidemia pelo HIV/aids entre UDI}

Como se pode depreender do acima exposto, a adequada compreensão das particularidades da população de usuários de drogas injetáveis e seus parceiros sexuais é imperiosa. Cabe desenvolver, de forma permanente, métodos apropriados e culturalmente sensíveis de monitoramento da infecção pelo HIV e demais patógenos de transmissão sangüínea e/ou sexual. A vigilância epidemiológica deve desempenhar um papel ativo. Também o conhecimento exaustivo dos fatores de risco, tanto sexuais como referentes ao uso de drogas, envolvidos na transmissão do HIV, assim como os marcadores de risco da infecção devem ser priorizados e avaliados, de maneira a fornecer subsídios para a avaliação dos programas de intervenção.

Até o presente momento, o Sistema de Notificação de Agravos (SINAN) para a aids representa o único banco de dados de caráter verdadeiramente nacional que permite monitorizar a epidemia do HIV/aids em nosso meio. Estes dados são úteis para avaliar a dinâmica da epidemia, ressalvandose o fato de que entre infecção pelo HIV e notificação de caso de aids decorrem cerca de dez anos, fazendo com que os casos notificados de aids representem apenas a ponta do iceberg da verdadeira extensão da infecção do HIV nesta e nas demais populações sob risco.

Os inquéritos de soroprevalência contribuem substancialmente para o dimensionamento da magnitude da epidemia, mas dizem respeito a indivíduos cuja data da infecção é desconhecida. Portanto, se mostram limitados quanto à sua capacidade de avaliar a efetiva dinâmica da epidemia. Os dados relativos à incidência do HIV são, sem dúvida alguma, os mais informativos com relação ao monitoramento do curso da epidemia. Entretanto, a notificação do HIV não é prática corrente e freqüentemente não é realizada em tempo ótimo, além da ausência nacional de estudos de coortes com acompanhamento sistemático de UDI, ao contrário do que vem 
sendo desenvolvido entre homens que fazem sexo com outros homens ${ }^{54}$. Ainda assim, os coeficientes de incidência obtidos a partir destes estudos são dificilmente generalizáveis, à medida em que a maioria destes estudos não inclui amostras representativas.

\section{Programas de redução de danos em UDI}

Os programas de redução de danos constituem a alternativa mais abrangente e bem sucedida de minimizar a transmissão do HIV e outros patógenos de transmissão sangüínea (e, em menor escala, de outras infecções de transmissão sexual). Sem que se entre em detalhe aqui na definição e constituição destes programas, ao que foi desenvolvido em momento anterior ${ }^{30}$, cabe mencionar que tais programas incluem um amplo conjunto de propostas preventivas hierarquizadas, conforme quadro abaixo e que apenas a desinformação os identifica unicamente com os Programas de Troca de Seringa (PTS).

Modelos hierárquicos relacionados ao uso de drogas e metas a atingir objetivando a interrupção da transmissão do HIV

Hierarchical models of drug-related goals and priorities to prevent HIV transmission

\begin{tabular}{cl}
\hline Nível & \multicolumn{1}{c}{ Metas } \\
\hline 1 & Prevenir o início do uso de drogas \\
2 & Abstinência de qualquer droga \\
3 & Abstinência de drogas injetáveis \\
4 & Usar equipamentos esterilizados \\
5 & Interromper o compartilhamento \\
6 & Usar de modo consistente e adequado \\
& o hipoclorito de sódio \\
\hline
\end{tabular}

Fonte: NRC. ${ }^{9}$ HIV Prevention: access to sterile needles, 1995.

É preciso ter em mente que estes programas de redução de danos estão longe de constituir uma panacéia e antes reduzem riscos do que os eliminam. Mesmo na Holanda, país que conta com um dos mais abrangentes conjuntos de iniciativas, precocemente implementados, estes programas encontram seu limite em níveis de transmissão do HIV e demais patógenos de transmissão sangüínea extremamente reduzidos para os padrões nacionais, mas ainda preocupantes ${ }^{55}$.

A experiência internacional combina experiências de epidemias abortadas no seu início, ditas "prevenidas"56 , e surtos epidêmicos em locais que contam com amplos recursos preventivos, como Vancouver, Canadá ${ }^{57}$.

Recente editorial da revista AIDS ${ }^{58}$ aponta para a necessidade de complementar os programas preventivos, ora em operação, com programas destinados a desestimular a transição das vias de auto-administração de drogas, de vias não-injetáveis para via injetáveis, diminuindo o contingente de "novos usuários injetáveis" que, como foi dito acima, estão sob intenso risco.

Até muito recentemente, os países da América Latina não contavam com iniciativas de redução de danos, que foram inicialmente introduzidas no continente em algumas regiões brasileiras na presente década ${ }^{59}$.

A despeito de inúmeras dificuldades, entre elas o permanente conflito entre as iniciativas de saúde pública e o aparato jurídico-policial, estes programas têm avançado. O Brasil conta hoje com 12 PTS em operação ${ }^{60}$, um número ainda reduzido dada a extensão da epidemia brasileira, as dimensões continentais do país e sua pronunciada heterogeneidade social.

Mais do que isso, dada a sua implementação tardia frente aos países, em que esses programas foram mais bem sucedidos, como a Austrália e a Holanda, os programas brasileiros terão de se haver com epidemias maduras, com altas taxas de infecção pelo HIV e demais patógenos de transmissão sangüínea, num desafio posto aos profissionais de saúde, governo e sociedade civil. 


\section{Summary}

Although limited in many ways, current HIVIAIDS knowledge provides information into the progression of the epidemic. Annual cases of AIDS according to the exposure categories reveal that the proportion of cases attributed to the injection drug use (IDUs) has steadily increased over the last 10 years in Brazil (21 percent in 1998). Given the growing role of this population in the transmission of the HIV, accurate knowlegde not only of the number of IDUs, but also their attitudes and practices, would be useful in allocating limited resources. Although improvements in estimating their number, e.g. capture-recapture methods as a reliable strategy, precise estimates remain difficult to be obtained. Also, HIV infected IDUs can cause a cascade of new infections, not only through needle sharing, but sexual or perinatal transmission. With respect to geographical variation, there is a cluster of the numbers of IDUs, but they are very dispersed across the country. The overall population of IDUs is quite heterogeneous, and their composition and characteristics are continually evolving with a marked change over time. In an effort to curb the rate of HIV infection among IDUs, 12 Syringe Exchange Programs (SEP) have provided consistent exchangeldistribution of new sterile needle/syringe in Brasil. However, comparative studies across programs are sparse, and need to be carried out. The present paper reviews the main findings of the HIV epidemiology and other blood-borne pathogens infection among IDUs, in Brazil and abroad, to foster the ongoing research and preventive initiatives. The use of combined data from different sources is encouraging. Comprehensive researches carried out in different settings, using data from distinct and complementary aggregation levels, under regular follow-up, are essential to monitor patterns and trends of the drug users population and drug consume patterns, especially the injection of illicit drugs. These data are essential to the implementation and permanent evaluation of preventive strategies, as well as tools for the optimal allocation of scarce resources. Due to the double risks to which IDUs are exposedparenteral and sexual - different infections are frequent in this population, taking place in an early moment of their drug use "career". Successful interventions must be precocious and comprehensive, providing counseling, testing for the different pathogens, and access to condoms and sterile injection equipment. Another core interventions set involves the prevention of the transition from noninjecting to injecting routes, and efforts to offer low-threshold and available anonymous treatment at no cost for either their clinical problems and harms secondary to drug abuse.

\section{Referências}

1. Ministério da Saúde. Coordenação Nacional de Doenças Sexualmente Transmissíveis e AIDS. Bol Epidemiol AIDS 1998; 11(1). [encarte].

2. Szwarcwald CL, Bastos Fl. Spatiotemporal model: an application to the AIDS epidemic in São Paulo, Brazil. In: Gierl L, Cliff AD, Valleron AJ, Farrington P, Bull M, editors. Geomed'97. Stuttgart:B.G.Teubner; 1998. p.43-53.

3. Bastos $\mathrm{FI}$, Coutinho K. A epidemia pelo HIV/AIDS entre usuários de drogas injetáveis (UDI) no Brasil: Cenários. In: A epidemia de AIDS no Brasil:situação e tendências. Brasília: Ministério da Saúde; 1997. p. 71-86.

4. Szwarcwald CL, Bastos Fl, Castilho EA. The dynamic of the AIDS epidemic in Brazil: a spatio-temporal analysis, 19871995. Braz J Infect Dis 1998;2(4):176-86.
5. Centers for Disease Control and Prevention. Update: syringe-exchange programs - United States, 1997. Morb Mortal Wkly Rep 1998;47:652-4.

6. Wodak A, Lurie P. A tale of two countries: attempts to control HIV among injecting drug users in Australia and the United States.J Drug Issues 1997; 27:117-34.

7. Feachem RGA.Valuing the past...investing in the future; evaluation of the National HIV/AIDS strategy 1993-94 to 1995-96. Canberra: Australian Government Publishing Service, Commonwealth Department of Human Services and Health; 1995.

8. UNAIDS \&WHO. Report on the global HIV/AIDS epidemic June 1998. Geneva; 1998 
9. Normand, Vlahov D, Moses LE, editors. Preventing HIV transmission: the role of sterile needles and bleach.Washington (DC): NRC National Academy Press; 1995.

10. Metzger DS, Woody GE, McLellan AT et al. Human immunodeficiency virus seroconversion among intravenous drug users in- and out-of-treatment. An 18month prospective follow-up.J AIDS 1993; 6:1049-56.

11. McCoy CB, Metch LR, Page JB et al. Injection druguser's practices and attitudes toward intervention and potential for reducing the transmission of HIV.Med Anthropol 1997; 18: 35-60.

12. Hubbard RL, Marsden ME, Cavanaugh E et al. Role of drug abuse treatment in limiting the spread od AIDS. Rev Infect Dis 1988; 10: 377-84.

13. Schoenbaum EE, Hartel DM, Gourevitch MN. Needle exchange use among a cohort of injecting drug users: description of methods. AIDS 1996; 19: 1729-34.

14. Vlahov D, Anthony JC, Muñoz A et al. The ALIVE Study: a longitudinal study of HIV-1 infection in travenous drug users: description of methods.J Drug Issues 1991; 21:759-76.

15. Carvalho HB, Bueno R. Prevalência da infecção pelo HIV e seus determinantes em sete cidades brasileiras - Projeto Brasil. São Paulo: 1997. [Relatório final apresentado ao Ministério da Saúde].

16. Telles PR, Bastos Fl, Guydish J et al. Risk behaviors and HIV seroprevalence among IDUs in Rio de Janeiro, Brazil. AIDS 1997; 11: S35-S44.

17. Carvalho HB, Mesquita F, Massad E et al. HIV and infections of similar transmission patterns in a drug injectors community of Santos, Brazil.J Acquir Immune Defic Syndr 1996; 12: 84-92.

18. Gawin FH, Kleber HD. Cocaine use in a treatment population: patterns and diagnostic distinctions. In: Kozel NJ, Adams EH.Cocaine use in America:epidemiologic and clinical perspectives. Washington (DC): NIDA; 1985. p. 18292. (NIDA Research Monograph, 61).

19. Anthony JC, Vlahov D, Nelson K et al. New evidence on intravenous cocaine use and the risk of infection with human immunodeficiency virus type 1. Am J Epidemiol 1991; 134:1175-89.

20. Turner CF, Miller HG, Moses LE. AIDS: sexual behavior and intravenous drug use. Washington (DC): National Academy Press; 1989.

21. Drucker E, Vermund SH. Estimating population prevalence of human immunodeficiency virus infection in urban areas with high rates of intravenous drug use: a model of the Bronx in 1988. Am J Epidemiol 1989; 130:133-42.

22. Abeni DD, Brancato G, Perucci CA. Capture-recapture to estimate the size of the population with HIV type 1 infection. Epidemiology 1994; 5:410-4.

23. Mastro TD, Kitayaporn D, Weniger BG et al. Estimating the number of HIV-infected injection drug users in Bangkok: a capture-recapture method. Am J Public Health 1994;84: 1094-9.
24. Caiaffa WT, Mingoti SA, Marques LF et al. Estimando a clientela dos projetos de redução de danos. O primeiro passo para estimar o número de usuários de drogas injetáveis infectados pelo HIV: resultados preliminares. In: IV Congresso Brasileiro de Epidemiologia - EPIRIO-98 1998; Rio de Janeiro.

25. Ministério da Saúde. Sociedade Civil Bem-Estar Familiar no Brasil - BEMFAM. Pesquisa Nacional sobre Demografia e Saúde. Brasília; 1996.

26. Carlini-Cotrim B, Carlini EA. O consumo de solventes e outras drogas em crianças e adolescentes de baixa renda na Cidade de São Paulo. Parte I: Estudantes de $1^{\circ}$ e $2^{\circ}$ graus da rede estadual. Rev ABP/APAL 1987; 9:49-58.

27. Carlini EA, coordenador.Consumo de drogas psicotrópicas no Brasil em 1987. Brasília: Centro de Documentação do Ministério da Saúde; 1989.

28. Carlini EA, Carlini-Cotrim B, Silva-Filho AR, Barbosa MTS. IILevantamento nacional sobre o uso de psicotrópicos em estudantes do $1^{\circ}$ e $2^{\circ}$ graus - 1989. São Paulo: Centro Brasileiro de Informações sobre Drogas Psicotrópicas CEBRID; 1990.

29. Galduróz JCF, D'Almeida V, Carvalho V, Carlini EA. IIILevantamento nacional sobre o uso de psicotrópicos em estudantes de $1^{\circ}$ e $2^{\circ}$ graus em 10 capitais brasileiras 1993. São Paulo: Centro Brasileiro de Informações sobre Drogas Psicotrópicas - CEBRID; 1993.

30. Bastos FI. Ruína \& reconstrução - AIDS e drogas injetáveis na cena contemporânea. Rio de Janeiro: Relume-Dumará/ ABIA/IMS-UERJ; 1996.

31. Barcellos C, Bastos FI. Redes sociais e difusão da AIDS no Brasil. Bol Oficina Sanit Panam 1996; 121:11-24.

32. Brown BS, Beschner GM. Handbook on risk of AIDS: injection drug users and sexual partners. Westport (CT): Greenwood Press; 1993.

33. WHO International Collaborative Group.Multi-city study on drug injecting and risk o HIV infection. Geneva:WHO; 1994.

34. Bastos Fl, Carlini-Cotrim B. O consumo de substâncias psicoativas entre os jovens brasileiros: dados, danos \& algumas propostas. In: Jovens acontecendo nas trilhas das políticas públicas. Brasília:Comissão Nacional de População e Desenvolvimento; 1998.v. 2, p. 645-69.

35. Hahn RA, Onorato IM, Jones S, Dougherty J et al. Prevalence of HIV infection among intravenous drug users in United States.J Am Med Assoc 1989;262:2677-84.

36. Nelson KE, Vlahov D, Solomon L et al.Temporal trends of incident HIV infection in a cohort of injectiong drug users in Baltimore, Maryland. Arch Intern Med 1995; 155:1305-11.

37. Friedman SR, Stepherson B, Woods J et al. Society, drug injectors, and AIDS.J Health Care Poor Underserved 1992; 3 : 73-89.

38. Friedman SR, Friedman P, Telles PR et al. New injectors and HIV-1 risk. In: Stimson G, Des Jarlais DC, Ball A, editors. Drug injecting and HIV infection: global dimensions and local responses. London:UCL Press; 1998. p.76-90. 
39. Schoenbaum EE, Hartel D, Selwyn PA et al. Risk factors for human immunodeficiency virus infection in intravenous drug users. N. Engl J Med 1989; 321:874-9.

40. Astemborski J, Vlahov D, Warren $L$ et al. The trading of sex for drugs or money and HIV seropositivity among female intravenous drug users. Am J Public Health 1994; 84:382-7.

41. Montoya I D, Atkinson JS. Determinants of HIV seroprevalence rates among sites participating in a community-based study of drug users. J Acquir Immune Defic Syndr Hum Retrovirol 1996; 13:169-76.

42. Greenberg J, Schnell D, Colon R. Behavior of crack cocaine users and their impact on early syphilis intervention. Sex Transm Dis 1992; 19:346-50.

43. Des Jarlais DC, Friedman SR. HIV and intravenous drug use. AIDS 1988; 2: S65-9.

44. Worth D. Self-help interventions with women at high risk of HIV infection. New York: Montefiore Medical Center; 1988.

45. Donoghoe MC. Sex, HIV and the injecting drug user. Br J Addictions 1992;87:405-16.

46. Padian NL, Shiboski E, Vittinghoff E, Hessol N. Heterosexual transission of HIV in Northern California: 1984-1993. Miami: Society for Epidemiological Research; 1994.

47. Hand IL, Wiznia RT, Checola MH et al. Human immunodeficiency virus seroposity in critically ill neonates in the South Bronx. Pediatr Infect Dis J 1992; 11:39-42.

48. Selwyn PA, Carter RJ, Schoenbaum EE et al. Knowledge of HIV antibody status and decision to continue or terminate pregnancy among intravenous drug users.J Am Med Assoc 1989; 261:3567-71.

49. Garfein RS, Vlahov D, Galai N et al.Viral infections in shortterm injection drug users: the prevalence of the hepatitis C, hepatitis B, human immuno-deficiency virus, and human T-lymphotropic viruses. Am J Public Health 1996;86: 655-61.

50. Strang J, Des Jarlais DC, Groffiths P, Gossop M. The study of transitions in the route of drug use: the route from one route to another. Br J Addictions 1992;87:473-84.
51. Van Ameijden EJC, van den Hoek JAR, Hartgers $C$ et al. Risk factors for the transition from noninjection drug use and accompanying AIDS risk behavior in a cohort of drug users. Am J Epidemiol 1994;139:1153-63.

52. Kalichman A. AIDS and intravenous drug use (IVDU) in Brazil. In: Monteiro MG, Inciardi JA, editors. Brasil - United States binational research. São Paulo:CEBRID; 1993.

53. Vlahov D, Brookmeyer RS. The evaluation of needle exchange programs. [Editorial]. Am J Public Health 1994; 84: 1889-91.

54. Sutmoller F, Souza CTV, Monteiro JC, Penna T.The Rio de Janeiro HIV vaccine site - I Recruitment strategies and sociodemographic data of a HIV negative homosexual and bisexual male cohort in Rio de Janeiro, Brazil. Mem Inst Oswaldo Cruz 1997;92:39-46.

55. Van Amejden EJC, Coutinho RA. Maximum impact of HIV prevention measures targeted at injectiong drug users. AIDS 1998; 12: 625-33.

56. Des Jarlais DC, Hagan H, Friedman SR et al. Maintaining low HIV seroprevalence in populations of injecting drug users. J Am Med Assoc 1995;274:1226-31.

57. Strathdee AS, van Ameijden E, Mesquita F et al. Can HIV epidemics among injection drug users be prevented? AIDS 1998; 12: S71-S79.

58. Des Jarlais DC. Understanding the long-term course of HIV epidemics [Editorial comment]. AIDS 1998; 12:669-70.

59. Marques F, Doneda D. A política brasileira de redução de danos à saúde pelo uso indevido de drogas: diretrizes e seus desdobramentos nos estados e municípios. In: Bastos $\mathrm{FI}$, Mesquita FC, editors. Troca de seringas, drogas e AIDS: ciência, debate e saúde pública. Brasília: Ministério da Saúde/UNDCP; 1998.

60. Minayo, MC, Deslandes SF, Souza ER et al. Avaliação das atividades de redução e danos no Brasil:relatóri final. Rio de Janeiro: FIOCRUZ/Ministério da Saúde: 1998. 